\title{
Clinical outcomes of laparoscopic surgery for advanced transverse and descending colon cancer: a single-center experience
}

\author{
Masashi Yamamoto · Junji Okuda • \\ Keitaro Tanaka $\cdot$ Keisaku Kondo $\cdot$ Nobuhiko Tanigawa • \\ Kazuhisa Uchiyama
}

Received: 26 August 2011/Accepted: 9 November 2011/Published online: 17 December 2011

(C) The Author(s) 2011. This article is published with open access at Springerlink.com

\begin{abstract}
Background The role of laparoscopic surgery in management of transverse and descending colon cancer remains controversial. The aim of the present study is to investigate the short-term and oncologic long-term outcomes associated with laparoscopic surgery for transverse and descending colon cancer.

Methods This cohort study analyzed 245 patients (stage II disease, $n=70$; stage III disease, $n=63$ ) who underwent resection of transverse and descending colon cancers, including 200 laparoscopic surgeries (LAC) and 45 conventional open surgeries (OC) from December 1996 to December 2010. Short-term and oncologic long-term outcomes were recorded.

Results The operative time was longer in the LAC group than in the OC group. However, intraoperative blood loss was significantly lower and postoperative recovery time was significantly shorter in the LAC group than in the OC group. The 5-year overall and disease-free survival rates for patients with stage II were $84.9 \%$ and $84.9 \%$ in the OC group and $93.7 \%$ and $90.0 \%$ in the LAC group, respectively. The 5-year overall and disease-free survival rates for patients with stage III disease were $63.4 \%$ and $54.6 \%$ in the OC group and $66.7 \%$ and $56.9 \%$ in the LAC group, respectively.

Conclusion Use of laparoscopic surgery resulted in acceptable short-term and oncologic outcomes in patients with advanced transverse and descending colon cancer.
\end{abstract}

M. Yamamoto $\cdot$ J. Okuda $\cdot$ K. Tanaka $\cdot$ K. Kondo

N. Tanigawa $\cdot$ K. Uchiyama $(\square)$

Departments of General and Gastroenterological Surgery,

Osaka Medical College Hospital, 2-7 Daigakumachi,

Takatsuki, Osaka 569-8686, Japan

e-mail: uchi@poh.osaka-med.ac.jp
Keywords Laparoscopic colon surgery - Colon cancer · Transverse colon cancer - Descending colon cancer . Survival rate

Since publication of the first report of laparoscopic surgery for colon cancer in 1991 [1], utilization of the procedure has steadily increased. Benefits of laparoscopic surgery relative to open surgery include improved cosmesis, improved short-term outcomes, reduced surgical trauma, reduced requirements for narcotic analgesia, earlier return of bowel function, and shorter postoperative hospital stay [2-4]. However, due to an insufficient body of clinical evidence, laparoscopic surgery for colon cancer has not yet replaced the conventional open surgery as the standard of care.

Although the safety and oncologic efficacy of laparoscopic surgery for treatment of colon cancer have been demonstrated in many randomized controlled trials [5-13], patients with transverse colon and descending colon cancer were excluded from many of these trials, mainly due to the difficulty in determining the appropriate operative procedure and the extent of lymphadenectomy [14]. Several recent studies have described the feasibility and safety of laparoscopic surgery for transverse and descending colon cancer [15-19]. However, there are few reports that describe the long-term outcomes associated with this management strategy.

In our institution, laparoscopic surgery was performed in more than 1,000 patients with colon cancer up to December 2008. Thus, the goal of this study is to investigate the shortterm and oncologic long-term outcomes associated with laparoscopic surgery for transverse and descending colon cancer. 


\section{Patients and methods}

The first laparoscopic resection for colon cancer at our institution was performed in 1996. At that time, laparoscopic colectomy was indicated only for early-stage cancer. Gradually, the indication for this procedure was expanded to more advanced stages of cancer. Further, with standardization of the surgical system, more than $90 \%$ of colorectal resections were ultimately performed laparoscopically. Conversion to conventional open surgery was performed at surgeon discretion. Between December 1996 and December 2008, 1,236 patients underwent surgery for colon cancer (laparoscopic surgery, $n=1,009$ : conventional open surgery, $n=227)$. Of these, 245 resections were performed for cancers of the transverse and descending colon without synchronous double malignancies. All patients underwent comprehensive assessment with blood testing, serum carcinoembryonic antigen measurement, colonoscopy, pathologic confirmation, barium or air enema, computed tomography (CT), and chest X-ray before surgery. If tumor localization was unclear, preoperative colonoscopic India ink tattooing and clipping was performed. The procedure for lymphadenectomy was determined based on depth of tumor invasion according to the Japanese Classification of Colorectal Carcinoma [20]. The laparoscopic nontouch isolation technique (i.e., the median-to-lateral approach) was utilized whenever possible. The study was approved by the institutional ethics of research committee, and informed consent was obtained from each patient.

\section{Study design}

This cohort study analyzed 245 patients (stage II disease, $n=70$; stage III disease, $n=63$ ) who underwent resection of the transverse and descending colon cancer, including 200 laparoscopic surgeries (LAC) and 45 conventional open surgeries (OC) from December 1996 to December 2010. Short-term outcomes and oncologic longterm outcomes were assessed among patients with stage II (70 cases) and stage III (63 cases) disease.

Laparoscopic procedures

For transverse colon lesions, proximal ligations of the right or left branch or the root of the middle colic vessels were conducted, and lymphadenectomy was performed simultaneously using the median-to-lateral approach. Mobilization was performed from the hepatic and/or splenic flexures. For the hepatic side, if the root of the middle colic vessels was clearly identified, the vein was divided just before the point at which it drained into the gastrocolic trunk of Henle.
For descending colon lesions, the left branch of the middle colic, left colic and sigmoid colic pedicles were identified, and lymphadenectomy was performed simultaneously with proximal ligations of the tumor-feeding vessels. The mesentery of the descending colon was gently mobilized from the ligament of Treitz by the median-tolateral approach. The omental bursa was entered, and the mesentery of the transverse colon was dissected from the inferior border of the pancreas. The bowel loop of transverse or descending colon was delivered under a wound protector through a 3- to 5-cm incision and was divided from the marginal vessels. The anastomosis was performed extracorporeally using the functional end-to-end method.

\section{Postoperative follow-up}

For follow-up, patients with stage I and II disease underwent assessment of serum carcinoembryonic antigen levels (at 3-month intervals during the first year and at 6-month intervals thereafter), chest and abdominopelvic CT (at 6-month intervals), and colonoscopy (at 1-year intervals) in addition to routine outpatient visits. Patients with stage III disease underwent assessment of serum carcinoembryonic antigen levels (at 4-month intervals during the first 2 years and at 6-month intervals thereafter), chest and abdominopelvic CT and colonoscopy at the same interval in addition to routine outpatient visits. Patients with stage III disease received adjuvant chemotherapy with 5-fluorouracil plus leucovorin per standards of care.

\section{Statistical analysis}

Statistical analysis was performed using JMP 8 (SAS Institute Inc., Cary, NC, USA) for Windows. Student's $t$ test, Mann-Whitney $U$ test and the $\chi^{2}$ test were used to compare continuous and categorical variables, respectively, with two-sided $p<0.050$ indicating significance. Patient survival analysis was performed using KaplanMeier survival curves with log-rank statistics.

\section{Results}

Laparoscopic surgery versus conventional open surgery

Patient demographics and pathologic variables are summarized in Table 1. Gender, age, body mass index (BMI), and American Society of Anesthesiology (ASA) classification were not significantly different when comparing the OC group and the LAC group. According to the tumornode-metastasis (TNM) classification, the proportion of patients with advanced stage was higher in the OC group than in the LAC group, mainly because LAC was initially 
Table 1 Characteristics of patients $(n=245)$ with transverse or descending colon cancer

\begin{tabular}{llll}
\hline & OC $(n=45)$ & $\begin{array}{l}\text { LAC } \\
(n=200)\end{array}$ & $p$ value $^{\mathrm{a}}$ \\
\hline Gender (male/female) & $26: 19$ & $110: 90$ & $0.734^{\mathrm{b}}$ \\
Age, years (mean, range) & $64(29-84)$ & $65(24-90)$ & 0.570 \\
BMI, kg/m & $21(16-34)$ & $22(16-32)$ & 0.102 \\
$\quad$ (mean, range) & & & \\
ASA classification & & 70 & $0.034^{\mathrm{b}}$ \\
I & 15 & 116 & \\
II & 20 & 14 & \\
III & 7 & 0 & $<0.001^{\mathrm{b}}$ \\
IV & 2 & & \\
Tumor classification & & 20 & \\
0 & 2 & 65 & \\
I & 2 & 55 & \\
II & 15 & 44 & \\
III & 19 & 16 & \\
IV & 7 & & \\
\hline
\end{tabular}

OC Conventional open surgery, $L A C$ laparoscopic surgery, $B M I$ body mass index, ASA American Society of Anesthesiologists

Clinical stage is classified by UICC-7 staging

a Student's $t$ test

b $\chi^{2}$ test

used only for early-stage cancers. Therefore, short-term outcomes and oncologic long-term outcomes were investigated in patients with stage II (70 cases) and stage III (63 cases) disease. Patient demographics and pathologic variables of these cases are summarized in Table 2. Patients with stage II disease undergoing OC included 3 right hemicolectomies, 4 left hemicolectomies, and 8 transverse colectomies, while patients with stage III disease undergoing OC included 1 right hemicolectomy, 6 left colectomies, and 12 transverse colectomies. By contrast, patients with stage II disease undergoing LAC included 15 right hemicolectomies, 21 left hemicolectomies, and 19 transverse colectomies, while patients with stage III disease undergoing LAC included 11 right hemicolectomies, 23 left colectomies, and 10 transverse colectomies. Five $(9.1 \%)$ patients with stage II disease required conversion to open surgery (bleeding, $n=3$; surgical technique, $n=1$; massive invasion, $n=1)$. Six (13.6\%) patients with stage III disease required conversion to open surgery (adhesion, $n=2$; massive invasion, $n=2$; bleeding, $n=1$; surgical technique, $n=1$ ). All patients underwent D3 lymphadenectomy according to the Japanese Classification of Colorectal Carcinoma [20]. Gender, age, BMI, ASA classification, tumor size, number of dissected lymph nodes, and tumor differentiation were not significantly different when comparing the OC group and the LAC group.
According to the TNM classification, the proportion of patients with pathologic $\mathrm{T}(\mathrm{pT})$ category was higher in the OC group than in the LAC group, likely because of the exclusion criteria utilized for this study. However, in terms of pathologic $\mathrm{N}(\mathrm{pN})$ category, there was no significant difference between the OC and the LAC group.

Table 3 presents the short-term outcomes of patients with stage II or stage III disease who underwent OC or LAC for transverse and descending colon cancer. The median operative time in patients with stage II disease was longer in the LAC group (230 min) than in the OC group (165 $\mathrm{min} ; p=0.012$ ), and the median operative time in patients with stage III disease was also longer in the LAC group (245 min) than in the OC group (202 min; $p=0.038$ ) with stage III. In patients with stage II disease, the median blood loss was significantly lower in the LAC group $(10 \mathrm{ml})$ than in the OC group $(100 \mathrm{ml} ; p<0.001)$, and in patients with stage III disease, the median blood loss was also significantly lower in the LAC group $(10 \mathrm{ml})$ than in the OC group $(155 \mathrm{ml} ; p<0.001)$. The duration until start of solid food after surgery was shorter in the LAC group ( 5 days) than in the OC group (7 days; $p=0.026$ ) in patients with stage II disease and was also shorter in the LAC group (4 days) than in the OC group (7 days; $p<0.001)$ in patients with stage III disease. The median hospital stay after surgery was shorter in the LAC group (15 days) than in the OC group (29 days; $p<0.001)$ in patients with stage II disease and was also shorter in the LAC group (7 days) than in the OC group (31 days; $p<0.001)$ in patients with stage III disease.

Table 4 summarizes the mortality and morbidity in each group. There were no perioperative deaths in patients with stage II disease. In patients with stage III disease, two patients died postoperatively: one from severe sepsis and septic shock in the LAC group, and one from liver failure with liver cirrhosis in the OC group. There was no significant difference in morbidity when comparing groups.

Table 5 summarizes the oncologic outcomes for the various groups. For patients with stage II disease, the median (range) follow-up period was 64 (10-154) months in the OC group and was 61 (12-128) months in the LAC group. For patients with stage III, the median (range) follow-up period was 53 (24-167) months in the OC group and 44 (9-145) months in the LAC group.

The 5-year overall and disease-free survival rates in patients with stage II disease were $84.9 \%$ and $84.9 \%$ in the OC group and $93.7 \%$ and $90.0 \%$ in the LAC group, respectively (Fig. 1A, B). The 5-year overall and diseasefree survival rates in patients with stage III disease were $63.4 \%$ and $54.6 \%$ in the OC group and $66.7 \%$ and $56.9 \%$ in the LAC group, respectively (Fig. 2A, B). The number of recurrences did not differ significantly between the LAC group and the OC group ( 2 versus $0 ; p=0.322$ ) in patients 
Table 2 Patient demographics and characteristics of transverse and descending colon cancer in patients with stage II or stage III disease

\begin{tabular}{|c|c|c|c|c|c|c|}
\hline & \multicolumn{3}{|l|}{ Stage II } & \multicolumn{3}{|l|}{ Stage III } \\
\hline & OC (15) & LAC (55) & $p$ value $^{\mathrm{b}}$ & OC (19) & LAC (44) & $p$ value $^{\mathrm{b}}$ \\
\hline Gender (male/female) & $9: 6$ & $27: 28$ & $0.452^{\mathrm{c}}$ & $9: 10$ & $22: 22$ & $0.848^{\mathrm{c}}$ \\
\hline Age, years ${ }^{\mathrm{a}}$ & $67(51-84)$ & $66(24-90)$ & 0.654 & $63(29-81)$ & $65(44-83)$ & 0.701 \\
\hline BMI, $\mathrm{kg} / \mathrm{m}^{2 \mathrm{a}}$ & $21(16-26)$ & $22(16-32)$ & 0.975 & $22(16-29)$ & $21(16-32)$ & 0.528 \\
\hline ASA classification & & & $0.353^{\mathrm{c}}$ & & & $0.470^{\mathrm{c}}$ \\
\hline I & 5 & 22 & & 7 & 18 & \\
\hline II & 8 & 29 & & 10 & 23 & \\
\hline III & 1 & 4 & & 1 & 3 & \\
\hline IV & 1 & 0 & & 1 & 0 & \\
\hline Tumor size, $\mathrm{cm}^{\mathrm{a}}$ & $5.4(2.5-7.6)$ & $4.8(1.4-8.7)$ & 0.316 & $5.0(3.2-11.2)$ & $4.2(1.0-10)$ & 0.119 \\
\hline Lymph nodes ${ }^{\mathrm{a}}$ & $19(7-27)$ & $15(3-33)$ & 0.132 & $14(5-41)$ & $16(5-35)$ & 0.711 \\
\hline pT category & & & $0.860^{\mathrm{c}}$ & & & $0.008^{\mathrm{c}}$ \\
\hline $\mathrm{T} 1$ & 0 & 0 & & 0 & 2 & \\
\hline $\mathrm{T} 2$ & 0 & 0 & & 1 & 5 & \\
\hline $\mathrm{T} 3$ & 14 & 52 & & 14 & 37 & \\
\hline $\mathrm{T} 4$ & 1 & 3 & & 4 & 0 & \\
\hline $\mathrm{pN}$ category & & & - & & & $0.566^{\mathrm{c}}$ \\
\hline N0 & 15 & 55 & & 0 & 0 & \\
\hline N1 & 0 & 0 & & 17 & 37 & \\
\hline $\mathrm{N} 2$ & 0 & 0 & & 2 & 7 & \\
\hline Tumor differentiation & & & $0.071^{\mathrm{c}}$ & & & $0.098^{\mathrm{c}}$ \\
\hline Well & 8 & 37 & & 9 & 18 & \\
\hline Moderate & 5 & 17 & & 7 & 25 & \\
\hline Poor & 2 & 0 & & 3 & 1 & \\
\hline Mucinous & 0 & 1 & & 0 & 0 & \\
\hline
\end{tabular}

Clinical stage is classified by UICC-7 staging

OC Conventional open surgery, LAC laparoscopic surgery, BMI body mass index, ASA American Society of Anesthesiologists, Well welldifferentiated adenocarcinoma, Moderate moderately differentiated adenocarcinoma, Poor poorly differentiated adenocarcinoma, Mucinous mucinous adenocarcinoma

Lymph nodes is number of lymph nodes removed

${ }^{\text {a }}$ Values expressed as median (range)

b Mann-Whitney $U$ test

c $\chi^{2}$ test

Table 3 Intraoperative and postoperative results of surgeries for transverse or descending colon cancer

\begin{tabular}{|c|c|c|c|c|c|c|}
\hline & \multicolumn{3}{|l|}{ Stage II } & \multicolumn{3}{|l|}{ Stage III } \\
\hline & OC (15) & LAC (55) & $p$ value $^{\mathrm{a}}$ & OC (19) & LAC (44) & $p$ value $^{\mathrm{a}}$ \\
\hline Operative time $(\mathrm{min})$ & $165(130-460)$ & $230(130-525)$ & 0.012 & $202(105-305)$ & $245(150-465)$ & 0.038 \\
\hline Blood loss (ml) & $100(40-660)$ & $10(10-1050)$ & $<0.001$ & $155(10-660)$ & $10(10-450)$ & $<0.001$ \\
\hline Days to diet & $7(5-34)$ & $5(2-22)$ & 0.026 & $7(4-34)$ & $4(3-36)$ & $<0.001$ \\
\hline Hospital stay (day) & $29(12-72)$ & $15(8-53)$ & $<0.001$ & $31(10-75)$ & $7(14-156)$ & $<0.001$ \\
\hline
\end{tabular}

$O C$ Conventional open surgery, $L A C$ laparoscopic surgery

Values expressed as median (range)

a Mann-Whitney $U$ test

with stage II disease or between the LAC group and the OC group (11 versus $7 ; p=0.346)$ in patients with stage III disease. There was no port-site recurrence or wound recurrence in either group, and there was no significant difference in the site of recurrence when comparing the groups. 
Table 4 Mortality and morbidity associated with surgery for transverse or descending colon cancer

\begin{tabular}{|c|c|c|c|c|c|c|}
\hline & \multicolumn{3}{|c|}{ Stage II } & \multicolumn{3}{|c|}{ Stage III } \\
\hline & $\begin{array}{l}\text { OC } \\
(15)\end{array}$ & $\begin{array}{l}\text { LAC } \\
(55)\end{array}$ & $p$ value $^{\mathrm{a}}$ & $\begin{array}{l}\text { OC } \\
(19)\end{array}$ & $\begin{array}{l}\text { LAC } \\
\text { (44) }\end{array}$ & $p$ value $^{\mathrm{a}}$ \\
\hline Mortality & 0 & 0 & - & 1 & 1 & 0.517 \\
\hline Morbidity & 5 & 11 & 0.069 & 6 & 7 & 0.163 \\
\hline SSI & 2 & 8 & & 2 & 6 & \\
\hline Leakage & 1 & 1 & & 2 & 1 & \\
\hline Ileus & 2 & 0 & & 1 & 0 & \\
\hline Colitis & 0 & 2 & & 0 & 0 & \\
\hline $\begin{array}{l}\text { Duodenal } \\
\text { ulcer }\end{array}$ & 0 & 0 & & 1 & 0 & \\
\hline
\end{tabular}

OC conventional open surgery, LAC laparoscopic surgery, Mortality within 30 days after surgery, SSI surgical-site infection

a $\chi^{2}$ test

Table 5 Five-year oncologic outcomes of patients who underwent surgery for transverse or descending colon cancer

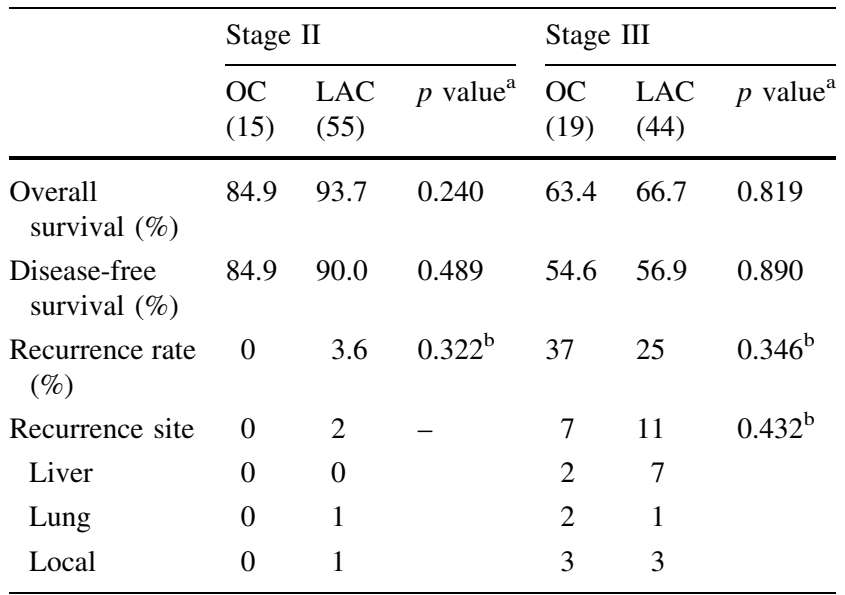

$O C$ conventional open surgery, LAC laparoscopic surgery, Recurrence site site of first recurrence

${ }^{\text {a }}$ Log-rank statistics

b $\chi^{2}$ test

\section{Discussion}

Several randomized controlled trials have demonstrated that laparoscopic surgery for colon cancer (excluding those with transverse or descending colon cancer) can achieve favorable short-term outcomes and oncologic outcomes that are similar to open surgery [5-13]. Other recent studies of laparoscopic surgery have also demonstrated the feasibility and safety of the procedure for transverse and descending colon cancers [15-19]. However, the oncologic outcomes of patients undergoing laparoscopic resection of transverse and descending colon cancer have not yet been studied.
A

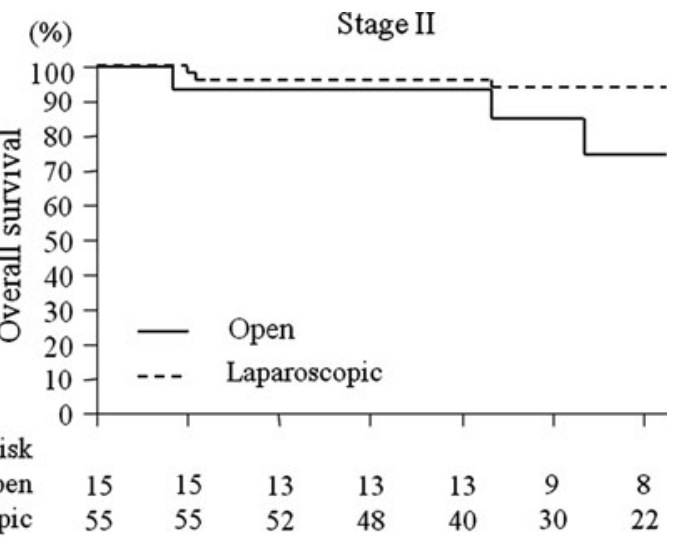

B

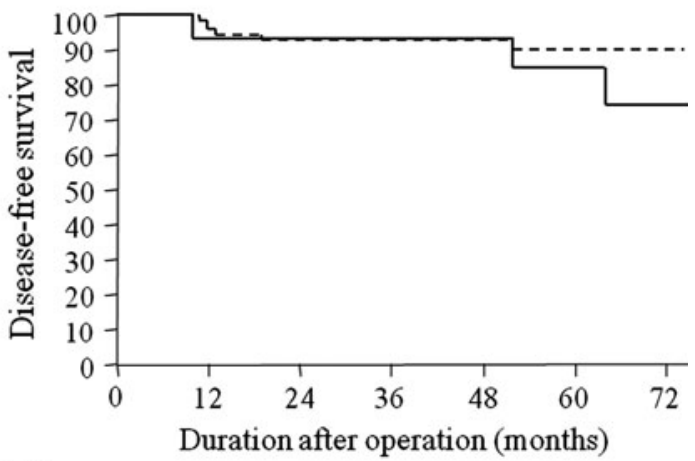

Number at risk

$\begin{array}{rrrrrrrr}\text { Open } & 15 & 15 & 13 & 13 & 12 & 9 & 8 \\ \text { Laparoscopic } & 55 & 54 & 50 & 48 & 39 & 29 & 21\end{array}$

Fig. 1 Kaplan-Meier curves of patients with stage II disease undergoing laparoscopic surgery or conventional open surgery: $\mathbf{A}$ overall survival rate and $\mathbf{B}$ disease-free survival rate. There was no statistically significant difference in survival between the two groups

Certainly, there are some difficulties when utilizing laparoscopic resection for transverse and descending colon cancer, as described in previous studies [15-19]; for example, mobilization, extent of resection, and details of lymphadenectomy may vary according to the precise location of the tumor in patients with transverse and descending colon cancer. In addition, resection of transverse and descending colon cancers that are adjacent to other critical structures, including the pancreas, duodenum, spleen, and the base of the mesenteric vessels, can result in major complications in case of dissection in the wrong plane. Therefore, thorough appreciation of the intricacies of venous anatomy at the gastrocolic trunk of Henle at the level of pancreas along the right plane is required when conducting this procedure. Jamali et al. [14] reported that a high-grade technique was required for splenic flexure mobilization, because of the requirement for extensive posterior dissection with simultaneous preservation of the vascular supply to the hind gut via the marginal artery as well as preservation of retroperitoneal structures, such as 
A
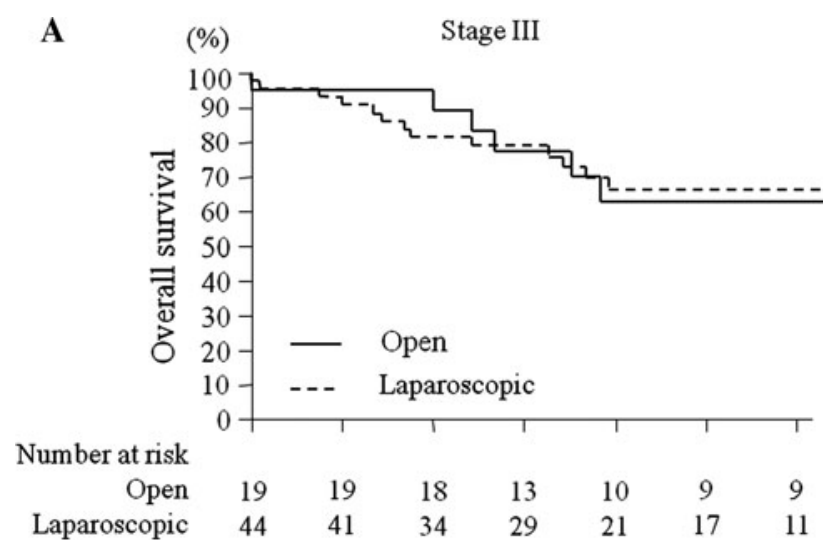

B

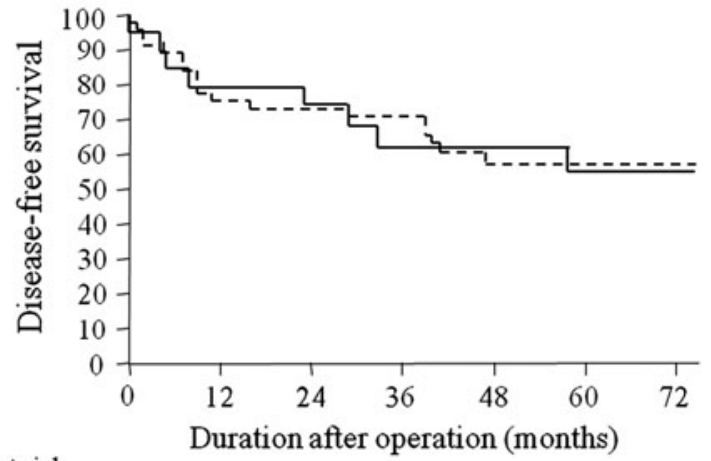

Number at risk

$\begin{array}{llllllll}\text { Open } & 19 & 16 & 14 & 10 & 10 & 8 & 8\end{array}$

$\begin{array}{llllllll}\text { Laparoscopic } & 44 & 34 & 33 & 28 & 20 & 16 & 11\end{array}$

Fig. 2 Kaplan-Meier curves of patients with stage II disease undergoing laparoscopic surgery or conventional open surgery: A overall survival rate and $\mathbf{B}$ disease-free survival rate. There was no statistically significant difference in survival between the two groups

the ureters and the tail of pancreas. Further, surgeons may have comparatively less experience in dealing with this procedure because the incidence of transverse and descending colon cancer is low. Thus, laparoscopic transverse colectomy and left colectomy are more difficult than sigmoid colectomy and right colectomy, which often limits their use for cancers of the transverse and descending colon, especially for those with advanced cancer. In our institution, laparoscopic surgery has been utilized in more than 200 patients with transverse and descending colon cancer. The present study characterized the short-term outcomes and oncologic long-term outcomes after resection for advanced cancer of transverse and descending colon in patients with stage II or stage III disease undergoing OC or LAC. Gender, age, BMI, ASA, and tumor size were similar in both groups. Operative time was longer in the LAC group than in the OC group, likely because of anatomical and technical difficulties. However, blood loss was significantly lower and the postoperative course of recovery was significantly shorter in the LAC group than in the OC group. The morbidity and mortality were not significantly different when comparing the two groups. Further, the number of dissected lymph nodes and the incidence of intraoperative injury were not significantly different when comparing the two groups, nor were there differences in the number of recurrences, overall survival, or disease-free survival. These data indicate that laparoscopic surgery for advanced transverse and descending colon cancer resulted in favorable short-term outcomes (i.e., lower blood loss, shorter postoperative stay) and similar oncologic long-term outcomes when compared with conventional open surgery. Thus, laparoscopic surgery is an acceptable management strategy for advanced colon cancer regardless of tumor location.

Successful laparoscopic surgery for transverse and descending colon cancer requires an advanced technique. Thus, acquisition of general laparoscopic skills is required to perform this fairly complex procedure. Since the number of patients requiring this specific procedure is relatively low, one way to gain this experience is through the development of laparoscopic skills when performing simpler, more common procedures, such as sigmoid colectomy and right colectomy. This experience may attenuate the otherwise steep learning curve needed to successfully achieve more complex laparoscopic procedures, thereby reducing the operative time, need for conversion to open procedures, and complication rate.

In conclusion, laparoscopic resection for transverse and descending colon cancer appears safe and feasible and produces acceptable short-term and oncologic long-term outcomes. Curative resection for advanced transverse and descending colon cancer is technically possible; however, the present data were derived from single-institution experience and were not generated in a prospective manner. Laparoscopic surgery for colon cancer has not yet replaced conventional open surgery as the standard, mainly because there is insufficient clinical evidence. Further, there are also controversies regarding the level of difficulty of the individual procedure, the lack of data regarding oncological long-term outcomes after curative resection, and hospital costs. However, the favorable results seen in several randomized controlled trials of the safety and oncologic efficacy of this procedure for advanced colon cancer have resulted in increased utilization of the procedure. Confirmation of the value of laparoscopic surgery for colon cancer in prospective randomized controlled trials may result in increased demand for laparoscopic procedures from physicians and patients. In our institution, the chief and senior surgeons are actively trained in laparoscopic colon surgery. Indeed, with standardization of the surgical system and gradual expansion of the indications, more than $90 \%$ of colon surgeries in 2010 were performed laparoscopically at our institution. Since the demand for laparoscopic surgery for colon cancer is expected to 
increase, chief and senior surgeons as well as young surgeons starting will gradually increase. Regardless, we believe that laparoscopic surgery may become the gold standard for management of colon cancer, regardless of stage or tumor location.

Disclosures Authors M. Yamamoto, J. Okuda, K. Tanaka, K. Kondo, N. Tanigawa, and K. Uchiyama have no conflicts of interest or financial ties to disclose.

Open Access This article is distributed under the terms of the Creative Commons Attribution Noncommercial License which permits any noncommercial use, distribution, and reproduction in any medium, provided the original author(s) and source are credited.

\section{References}

1. Jacobs M, Verdeja JC, Goldstein HS (1991) Minimally invasive colon resection (laparoscopic colectomy). Surg Laparosc Endosc $1: 144-150$

2. Schwenk W, Haase O, Neudecker J, Müller JM (2005) Short term benefits for laparoscopic colorectal resection. Chochrane Database Syst Rev 20(3):CD003145

3. Harmon GD, Senegore AJ, Kilbride MJ, Warzynski MJ (1994) Interleukin-6 response to laparoscopic and open colectomy. Dis Colon Rectum 37:754-759

4. Nishiguti K, Okuda J, Toyoda M, Tanaka K, Tanigawa N (2001) Comparative evaluation of surgical stress of laparoscopic and open surgeries for colorectal carcinoma. Dis Colon Rectum 44:223-230

5. Lacy AM, Gracia-Valdecasas JC, Delgango S, Castells A, Taura P, Pique JM, Visa J (2002) Laparoscopy-assisted colectomy versus open colectomy for treatment of non-metastatic colon cancer: a randomized trial. Lancet 359:2224-2229

6. Hazebroec EJ, Colon study group (2002) A randomized clinical trial comparing laparoscopic and open resection for colon cancer. Surg Endsc 16:949-953

7. Leung KL, Kwok SP, Lam SC, Lee JF, Yiu RY, Ng SS, Lai PB, Lau WY (2004) Laparoscopic resection of rectosigmoid carcinoma: prospective randomized trial. Lancet 363:1187-1192

8. Clinical Outcomes of Surgical Therapy Study Group (2004) A comparison of laparoscopically assisted and open colectomy for colon cancer. N Engl J Med 350:2050-2059
9. Braga M, Frasson M, Zuliani W, Civelli V, Di Carlo V (2005) laparoscopic vs. open colectomy in cancer patients: long term complications, quality of life, and survival. Dis Colon Rectum 48:2217-2223

10. Jayne DG, Guillou PJ, Thorpe H, Quirke P, Copeland J, Smith AM, Heath RM, Brown JM (2007) Randomized trial of laparoscopic-assisted resection of colorectal carcinoma:3-year result of UK MRC CLASSICC trial group. J Clin Oncol 25:3061-3068

11. Clinical Outcomes of Surgical Therapy Study Group (2007) Laparoscopic colectomy for cancer is not inferior to open surgery based on 5-year data from the COST study group trial. Ann Surg 246:655-664

12. The Colon Cancer Laparoscopic or Open Resection Study Group (2009) Survival after laparoscopic surgery versus open surgery for colon cancer: long-term outcome of a randomized clinical trial. Lancet Oncol 10:44-52

13. Jayne DG, Thorpe HC, Copeland J, Quirke P, Brown JM, Guillou PJ (2010) Five-year follow-up of the medical research council CLASICC trial of laparoscopically assisted versus open surgery for colorectal cancer. Br J Surg 97:1638-1645

14. Jamali FR, Soweid AM, Dimassi H, Bailey C, Leroy J, Marescaux J (2008) Evaluating the degree the difficulty of laparoscopic colorectal surgery. Arch Surg 143(8):762-767

15. Schlachta CM, Mamazza J, Poulin EC (2007) Are transverse colon cancers suitable for laparoscopic resection? Surg Endosc 21:396-399

16. Lee YS, Lee IK, Kang WK, Cho HM, Park JK, Oh ST, Kim JG, Kim YH (2008) Surgical and pathological outcome of laparoscopic surgery for transverse colon cancer. Int J Colorectal Dis 23:669-673

17. Yamamoto S, Fujita S, Akasu T, Yamaguchi T, Moriya Y (2009) Laparoscopic surgery for transverse and descending colon carcinomas has comparable safety to laparoscopic surgery for colon carcinomas at other sites. Dig Surg 26:487-492

18. Zmora O, Bar-Dayan A, Khaikin M, Lebeydev A, Shabtai M, Ayalon A, Rosin D (2010) Laparoscopic colectomy for transverse colon cancer. Tech Coloproctol 14:25-30

19. Akiyoshi T, Kuroyanagi H, Fujimoto Y, Konishi T, Ueno M, Oya M, Yamaguchi T (2010) Short-term outcomes of laparoscopic colectomy for transverse colon cancer. J Gastrointest Surg $14: 818-823$

20. Japanese Society for Cancer of the Colon and Rectum (2009) Guidelines for Therapy of Colorectal Cancer. Kanehara Shuppan, Tokyo 